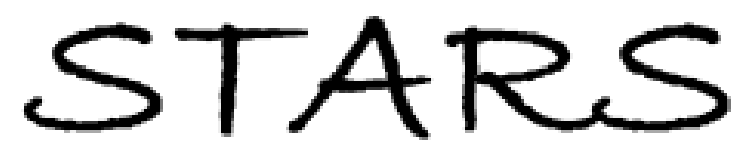

University of Central Florida

STARS

$1-1-2007$

\title{
Single glass substrate liquid crystal device using electric field- enforced phase separation and photoinduced polymerization
}

Hongwen Ren

University of Central Florida

Shin-Tson Wu

University of Central Florida

Yi-Hsin Lin

Find similar works at: https://stars.library.ucf.edu/facultybib2000

University of Central Florida Libraries http://library.ucf.edu

This Article is brought to you for free and open access by the Faculty Bibliography at STARS. It has been accepted for inclusion in Faculty Bibliography 2000s by an authorized administrator of STARS. For more information, please contactSTARS@ucf.edu.

\section{Recommended Citation}

Ren, Hongwen; Wu, Shin-Tson; and Lin, Yi-Hsin, "Single glass substrate liquid crystal device using electric field-enforced phase separation and photoinduced polymerization" (2007). Faculty Bibliography 2000s.

7568.

https://stars.library.ucf.edu/facultybib2000/7568

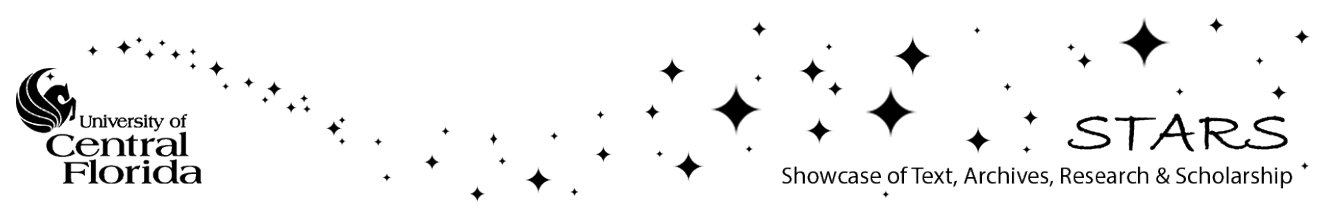




\section{Single glass substrate liquid crystal device using electric field-enforced phase separation and photoinduced polymerization}

Cite as: Appl. Phys. Lett. 90, 191105 (2007); https://doi.org/10.1063/1.2737366

Submitted: 24 March 2007 . Accepted: 13 April 2007 . Published Online: 07 May 2007

Hongwen Ren, Shin-Tson Wu, and Yi-Hsin Lin

ARTICLES YOU MAY BE INTERESTED IN

Single-substrate cholesteric liquid crystal displays by colloidal self-assembly Applied Physics Letters 88, 043502 (2006); https://doi.org/10.1063/1.2167398

Network morphology of polymer stabilized liquid crystals

Applied Physics Letters 71, 2454 (1997); https://doi.org/10.1063/1.120087

Fast-switching initially-transparent liquid crystal light shutter with crossed patterned electrodes

AIP Advances 5, 047118 (2015); https://doi.org/10.1063/1.4918277
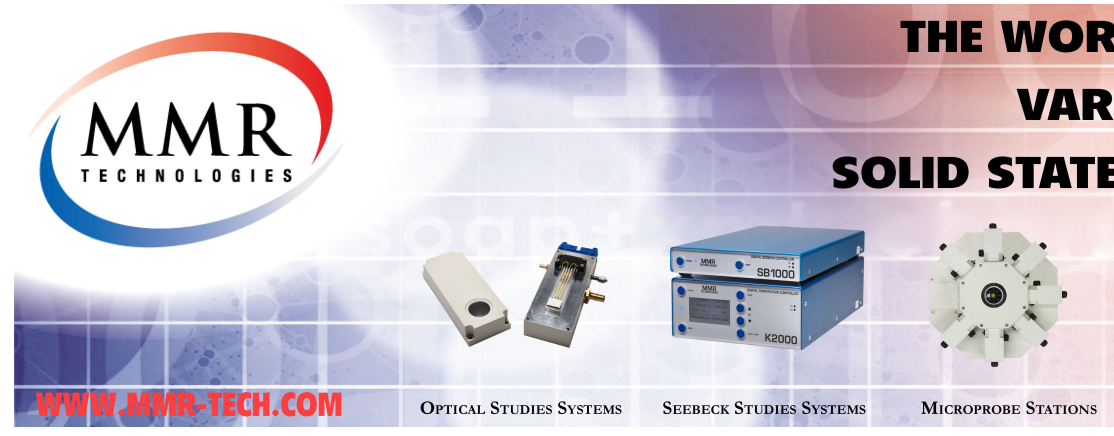

THE WORLD'S RESOURCE FOR VARIABLE TEMPERATURE

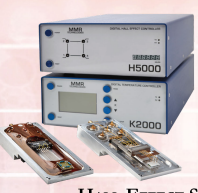

Hall Effect Study Systems and Magnets 


\title{
Single glass substrate liquid crystal device using electric field-enforced phase separation and photoinduced polymerization
}

\author{
Hongwen Ren and Shin-Tson $\mathrm{Wu}^{\mathrm{a}}$ \\ College of Optics and Photonics, University of Central Florida, Orlando, Florida 32816 \\ Yi-Hsin Lin \\ Department of Photonics, Institute of Electro-Optical Engineering, National Chiao Tung University, \\ Hsinchu 30010, Taiwan
}

(Received 24 March 2007; accepted 13 April 2007; published online 7 May 2007)

\begin{abstract}
An in-plane switching liquid crystal (LC) cell using a glass substrate and a photoinduced polymer layer is demonstrated. The fabrication process is based on the electrodynamics of dielectric fluids. When the fringing field is present, the LC molecules tend to aggregate in the strong electric field regions while the monomers diffuse to the weak field regions. After photopolymerization, the LC molecules are confined by a thin polymer layer and polymer walls which define the cell gap. This approach enables single-substrate large panel display devices to be fabricated. (C) 2007 American Institute of Physics. [DOI: 10.1063/1.2737366]
\end{abstract}

Liquid crystals (LCs) have been widely used for displays, ${ }^{1}$ phase modulators, ${ }^{2,3}$ adaptive-focus lens, ${ }^{4,5}$ and many other optical components. Due to the fluidic nature, usually LC molecules are confined between two glass substrates. Recently, LC devices using only one glass substrate have been demonstrated in an in-plane switching (IPS) cell. ${ }^{6-9}$ The reasons for choosing IPS cells are obvious: (1) the interdigitated electrodes are in the same substrate which can be a glass or plastic substrate overcoated with a LC alignment layer, and (2) the device exhibits a wide viewing angle. Compared to conventional two glass substrate LC devices, the single substrate LC devices are attractive because of their lighter weight and potentially lower cost.

A single-substrate IPS cell was first demonstrated using photoenforced anisotropic phase separation between LC and prepolymer. ${ }^{6}$ After photopolymerization, the prepolymer forms a thin layer which is floated on the LC surface. The stability of the device is a concern due to the lack of a strong solid support to bear the weight of the polymer layer. To overcome this problem, a two-step photopolymerization process was developed. ${ }^{8}$ The first step is to selectively expose monomers through a photomask for forming polymer walls. The second step is to remove the photomask and expose the uncured monomers so that they could undergo anisotropic polymerization. Although these devices possess a good mechanical stability, this approach encounters two problems. Firstly, an error contraposition of the photomask would cause the formed polymer walls to occupy the active regions. Secondly, a high resolution photomask can severely diffract light which, in turn, widens the polymer walls during phase separation and decreases the aperture ratio of the active pixels.

Recently, our group developed an IPS LC cell using a single glass substrate and an anisotropic polymer film. ${ }^{9}$ This cell exhibits almost the same electro-optical properties as that of a conventional IPS LC cell using two glass substrates. However, this kind of LC cell also suffers from weak mechanical stability because it has no spacers to support the soft polymer film. Therefore, it is difficult to control the uniformity while making a large LC panel.

\footnotetext{
${ }^{a)}$ Electronic mail: swu@ mail.ucf.edu
}

In this letter, we demonstrate a single-substrate IPS cell based on electric field-enforced anisotropic phase separation between the LC and the liquid monomer. Using this approach, the LC and monomer mixture can be phase separated on a substrate surface. After photopolymerization, both polymer walls and top polymer layer are formed simultaneously. Such a LC device possesses a good mechanical stability which enables the fabrication of large panels with high resolution. Moreover, the fabrication process is fairly simple.

Electric field-induced anisotropic phase separation was first demonstrated in reflective displays employing two glass substrates. ${ }^{10}$ In this approach, the polymer in the cell was distributed in the interpixel regions working as walls. However, in our device a portion of the polymers are used as walls and the majority are used as top cover layer to prevent the LC from leaking.

Figures 1(a)-1(d) illustrate the fabrication procedures of our LC cell. A glass substrate with interdigitated indium tin oxide (ITO) electrodes was chosen as a bottom substrate. The ITO glass was overcoated with a thin polyimide alignment layer and buffed along the ITO strip direction. A mixture of LC and photocurable monomer was spin coated over

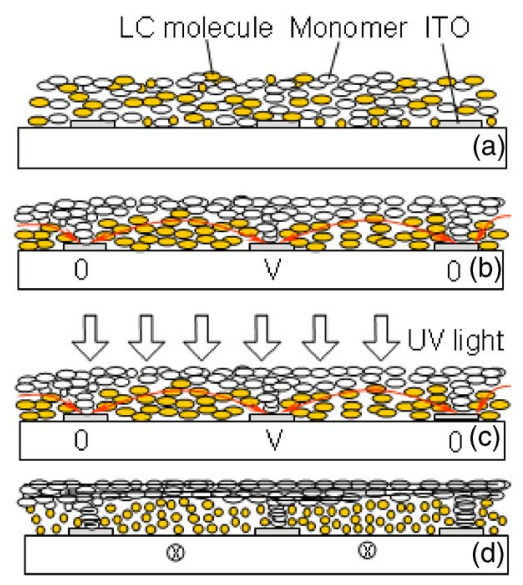

FIG. 1. (Color online) Procedures for fabricating a single glass substrate IPS cell: (a) coating film on the substrate surface, (b) applying a voltage to the ITO strips, (c) UV curing, and (d) removing the voltage. 
the substrate surface, as shown in Fig. 1(a). When a voltage is applied to the ITO strips, the dielectric fluid experiences an electric field and bears a force. The force can be expressed as $^{11}$

$$
\mathbf{F}=\mathbf{P} \cdot \nabla \mathbf{E},
$$

where $\mathbf{P}$ is the polarization of dielectric material and $\mathbf{E}$ the electric field strength. Further, $\mathbf{P}$ is related to the electric field as

$$
\mathbf{P}=\varepsilon_{0}(\varepsilon-1) \mathbf{E},
$$

where $\varepsilon_{0}$ is the permittivity of free space and $\varepsilon(>1)$ represents the dielectric constant of the LC or the liquid monomer. Since the LC employed has a larger dielectric constant than the liquid monomer, the $\mathrm{LC}$ molecules will experience a greater force from the electric field. Under such a circumstance, the LC molecules would drift toward the high electric field regions while the monomers aggregate in the weak electric field regions, as Fig. 1(b) shows. In an IPS cell, the weakest electric field is in the centers of ITO electrodes. After phase separation, the liquid monomers are cured by an UV light, as shown in Fig. 1(c). After polymerization, both polymer layer and polymer walls are formed at the same time. The polymer layer covers the active $\mathrm{LC}$ regions, while the polymer walls sit on top of ITO electrodes, as shown in Fig. 1(d). In a real display panel, these inactive regions are covered by the absorbing black matrices for improving contrast ratio. When the voltage is removed, the LC molecules are reoriented along the rubbing direction due to the anchoring effect of the alignment layer. As indicated in Fig. 1(d), the rubbing direction of the bottom substrate is pointing into the paper.

Based on the above mechanism, we fabricated an IPS LC cell with a single glass substrate. The ITO electrode on the bottom substrate surface was etched to form interdigitated chevron shapes in order to create multiple domain structures for widening the viewing angle. The angle of the zigzag ITO strips is $150^{\circ}$. The width of the electrode strip is $4 \mu \mathrm{m}$ and the electrode gap is $10 \mu \mathrm{m}$. The surface of the substrate was coated with a thin polyimide layer and buffed in one direction at $15^{\circ}$ with respect to the electrode strips. A mixture consisting of $55 \mathrm{wt} \%$ nematic LC (E7, Merck $\varepsilon_{\mathrm{LC}}$ $\sim 12$ ) and 45 wt $\%$ photocurable monomer NOA65 (Norland Adhesive $\varepsilon_{\mathrm{p}} \sim 5$ at $f=1 \mathrm{kHz}$ ) was spin coated (at $\sim 4000 \mathrm{rpm}$ for $15 \mathrm{~s}$ ) on a glass substrate at room temperature $\left(T \sim 21^{\circ} \mathrm{C}\right)$. The volume of the $\mathrm{LC}$ is approximately the same as that of the monomer because E7 has a slightly higher density than NOA65. An electric field of $5 V_{\text {rms }} / \mu \mathrm{m}$ was applied to the ITO strips for $1 \mathrm{~min}$. Afterwards, the mixture was exposed to an UV light $(\lambda=365 \mathrm{~nm})$ at intensity of $\sim 35 \mathrm{~mW} / \mathrm{cm}^{2}$ for $15 \mathrm{~min}$.

After polymerization the cell is transparent in the voltage-off state. To observe the phase separation result, we placed the sample under a polarizing optical microscope with a white light lamp. The axes of the polarizer and analyzer are crossed. When the sample was rotated along the azimuthal direction, the transmitted light intensity changed correspondingly. When the rubbing direction of the IPS cell was oriented parallel to the optic axis of the entrance polarizer, a dark state was obtained, as shown in Fig. 2(a). Figure 2(b) shows the on-state transmittance of the IPS cell at $V=20 V_{\text {rms. }}$. The chevron structures are clearly observed. The black grids indicate that the polymer walls formed on the

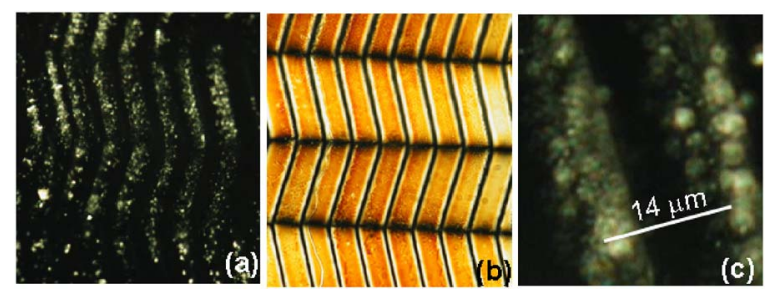

FIG. 2. (Color online) Microscope photos of the IPS LC cell: (a) $V=0$, (b) $V=20 V_{\text {rms }}$, and (c) magnified image of the top polymer layer.

middle of ITO strips are isotropic. The width of the polymer wall is about $2 \mu \mathrm{m}$ which is less than the width $(4 \mu \mathrm{m})$ of the ITO strips. In in-plane switching, the fringing field is inhomogeneous and the weakest field occurs at the middle of the ITO electrodes. These polymer walls function as spacers for controlling the cell gap and supporting the top polymer layer. Due to the polymer networks, the IPS cell should have a good mechanical stability and uniform cell gap.

The electro-optical properties of the LC cell were studied by measuring the transmittance of the cell using a $\mathrm{He}-\mathrm{Ne}$ laser $(\lambda=633 \mathrm{~nm})$ beam. The cell was placed between two crossed polarizers. The transmitted light was detected by a photodiode set at $20 \mathrm{~cm}$ away from the sample. The response time of the cell was recorded by a digital oscilloscope. The voltage dependent transmittance was collected by a LABVIEW data acquisition system.

Figure 3 shows the voltage dependent transmittance of the IPS cell. At $V=0$, a fairly good black state is observed. As the applied voltage exceeds $5 V_{\text {rms }}$, the transmission begins to increase indicating that the LC directors are reoriented by the electric field. At $20 V_{\text {rms }}$, the transmission reaches a maximum. Continue to increase voltage leads to a decreased transmittance. This is because the total phase retardation of the LC cell exceeds $1 \pi$.

The contrast ratio was measured to be $\sim 200: 1$. For comparison, the contrast ratio of a conventional IPS cell using two glass substrates exceeds 500:1. By a large, the bottom alignment layer still aligns the bulk LC directors reasonably well. At a closer look on Fig. 2(a), we find a weak light leakage in the LC-rich regions. To investigate what causes this light leakage, we zoomed into the top polymer layer of the LC cell. As shown in Fig. 2(c), some LC bubbles with $2-3 \mu \mathrm{m}$ in size are kept within the top polymer layer. These results imply that the LC and monomer are not completely phase separated so that a small amount of LC bubbles are retained in the polymer matrices. These micron-sized LC droplets cause light leakage between crossed polarizers and a little bit of light scattering. As a result, the device contrast ratio is degraded.

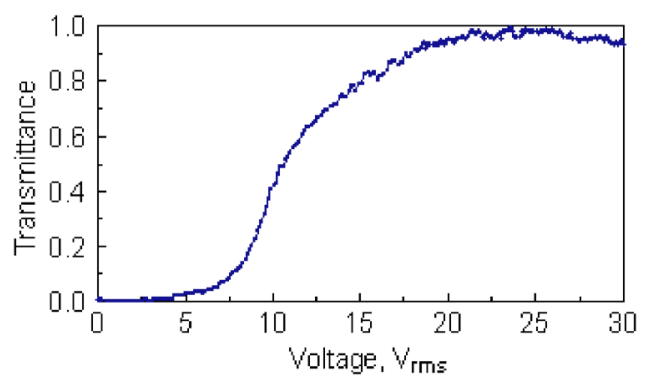

FIG. 3. (Color online) Voltage dependent transmittance of the IPS cell: LC employed is E7 and $\lambda=633 \mathrm{~nm}$. 


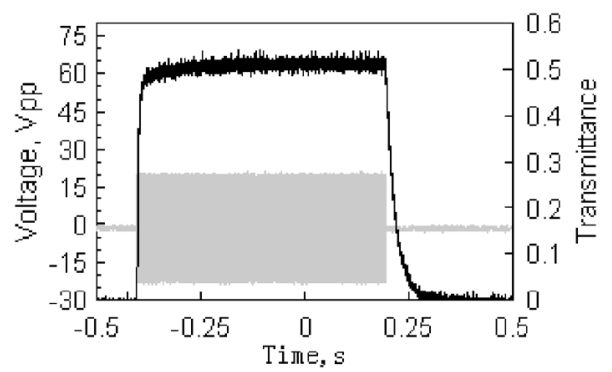

FIG. 4. Measured response times of the IPS cell: rise time $\sim 10 \mathrm{~ms}$ and decay time $\sim 75 \mathrm{~ms}$.

The response time of the IPS cell was measured using a square voltage burst at $f=1 \mathrm{kHz}$ and $20 V_{\text {rms }}$. Results are shown in Fig. 4. The measured rise and decay times are $\sim 10$ and $\sim 75 \mathrm{~ms}$, respectively. In a vertical alignment or homogeneous LC cell, the optical decay time $\left(\tau_{\text {decay }}\right.$ which is defined as $100 \%-10 \%$ transmittance change) is related to the LC directors decay time $\left(\tau_{0}\right)$ as follows: ${ }^{12}$

$$
\tau_{\text {decay }}=\frac{\tau_{0}}{2} \ln \left(\frac{\delta_{o} / 2}{\sin ^{-1}\left(\sqrt{0.1} \sin \left(\delta_{o} / 2\right)\right)}\right) .
$$

In Eq. (3), $\tau_{0}=\gamma_{1} d^{2} / k_{22} \pi^{2}$ is the LC director relaxation time where $\gamma_{1}$ is the rotational viscosity, $d$ is the LC cell gap, $k_{22}$ is the twist elastic constant, and $\delta_{0}$ is the initial phase of the LC cell which is usually $1 \pi$. If we neglect the logarithmic term in Eq. (3), we find $\tau_{\text {decay }} \sim \tau_{0} / 2$. In our LC cell, $\gamma_{1}$ and $k_{22}$ are $0.25 \mathrm{~Pa} \mathrm{~s}$ and $8.8 \mathrm{pN}$, respectively. From Eq. (3), $d$ is estimated to be $\sim 7.2 \mu \mathrm{m}$. Because the monomer NOA65 and E7 have about the same volume, the thickness of the top polymer layer is $\sim 7 \mu \mathrm{m}$.

The E7 IPS cell we fabricated is for proving concept only because its response time is too slow for practical applications. Two factors causing the observed slow response time are thick cell gap and high viscosity. In reality, the thin film transistor-grade LC mixture has a two to three times lower viscosity than E7 and the cell gap is about $4 \mu \mathrm{m}$. To make the cell gap thinner while retaining a robust top polymer layer and rigid polymer walls, we should decrease the $\mathrm{LC}$ concentration in the $\mathrm{LC} /$ monomer mixture. A thinner LC cell improves not only response time but also molecular alignment because the influence of bottom alignment layer to the bulk LC molecules is stronger. Better LC alignment would result in a higher contrast ratio.

In conclusion, we have demonstrated a single glass substrate LC cell using electric field-enforced stratification method. Such a LC cell exhibits advantages in simple fabrication, lightweight, and good mechanical stability. This approach opens a possibility for making single-substrate large panel display devices.

The authors would like to thank H. Xianyu and S. Gauza for their experimental assistance and useful discussions.

${ }^{1}$ S. T. Wu and D. K. Yang, Reflective Liquid Crystal Displays (Wiley, New York, 2001).

${ }^{2}$ D. P. Resler, D. S. Hobbs, R. C. Sharp, L. J. Fridman, and T. A. Dorschner, Opt. Lett. 21, 689 (1996).

${ }^{3}$ R. L. Sutherland, L. V. Natarajan, V. P. Tondiglia, and T. J. Bunning, Chem. Mater. 5, 1533 (1993).

${ }^{4}$ S. Sato, Jpn. J. Appl. Phys. 18, 1679 (1979).

${ }^{5}$ H. Ren, Y. H. Fan, S. Gauza, and S. T. Wu, Appl. Phys. Lett. 84, 4789 (2004).

${ }^{6}$ V. Vorflusev and S. Kumar, Science 283, 1903 (1999).

${ }^{7}$ I. Kim, J. H. Kim, D. Kang, D. M. A. Kooijman, and S. Kumar, J. Appl. Phys. 92, 7699 (2002).

${ }^{8}$ R. Penterman, S. T. Klink, H. de Koning, G. Nisato, and D. J. Broer, Nature (London) 417, 55 (2002).

${ }^{9}$ Y. H. Lin, H. Ren, S. Gauza, Y. H. Wu, Y. Zhao, J. Y. Fan, and S. T. Wu, J. Disp. Technol. 2, 21 (2006).

${ }^{10}$ Y. Kim, J. Francl, B. Taheri, and J. L. West, Appl. Phys. Lett. 72, 2253 (1998).

${ }^{11}$ P. Penfield and H. A. Haus, Electrodynamics of Moving Media (MIT, Cambridge, 1967).

${ }^{12}$ H. Wang, T. X. Wu, X. Zhu, and S. T. Wu, J. Appl. Phys. 95, 5502 (2004). 